\title{
Endoscopic Treatment of Piriformis Syndrome Results in a Significant Improvement in Pain Visual Analog Scale Scores
}

\author{
Frédérique Vanermen, M.D., and Jan Van Melkebeek, M.D.
}

\begin{abstract}
Purpose: To survey the clinical satisfaction of patients who have had an endoscopic piriformis release with a sciatic nerve neurolysis. Methods: Patients with piriformis syndrome (PS) who were treated with an endoscopic piriformis release combined with a sciatic neurolysis between 2013 and 2018 were identified. All patients had a minimum follow-up of 1 year. Patients were contacted by telephone in 201,9 whereby the rate of satisfaction and pain were evaluated with the Benson surgery scale and the visual analog scale (VAS). Results: The average duration of symptoms at first presentation was 34 months (range 16-54 months). Thirty-seven cases (82,2\%) had an EMG that showed sciatic compression around the piriformis muscle. The average preoperative VAS pain score was $7.4( \pm 0.8)$. The mean VAS score at the time of the survey was $1.9( \pm 2.4)$. The difference in median preoperative and postoperative VAS was 6 . Using the Wilcoxon signedrank test, we found there was a significant improvement of VAS score (with $P<.001$ ). Using the Benson operative scale, we found that outcomes at the time of the survey were excellent in 23 patients ( 24 cases, $53.4 \%)$, good in $10(22.2 \%)$, fair in $3(6.7 \%)$, and poor in $8(17.7 \%)$. Three patients with a poor result had a wrong diagnosis of PS and ended up needing treatment for a different diagnosis. In total, 33 patients answered affirmative on the question if they would undergo the procedure again. Apart from 3 local wound infections, no major complications were observed. Conclusions: Once a diagnosis is made, the endoscopic release of the piriformis tendon results in significant improvement in Pain VAS scores for patients with refractory symptoms despite conservative treatment. Level of Evidence: Level IV, therapeutic case series.
\end{abstract}

$\mathbf{T}$ he piriformis muscle is located within the subgluteal space and lies in direct contact with the sciatic nerve. ${ }^{1}$ Piriformis syndrome (PS) is a pathology in which the sciatic nerve is compressed by the piriformis muscle on the course of its passage through the subgluteal space, causing pain and disturbances in the gluteal region with possible radiation toward the posterior thigh. ${ }^{2}$

Robinson ${ }^{3}$ introduced the term "piriformis syndrome" in 1947 and suggested 6 main features of the pathology: (1) history of trauma in the gluteal or sacroiliac region; (2) pain in the sacroiliac joint, greater sciatic notch and

From Orthopaedic Centre Antwerp (ORTHOCA), Antwerp, Belgium.

The authors report that they have no conflicts of interest in the authorship and publication of this article. Full ICMJE author disclosure forms are available for this article online, as supplementary material.

Received February 22, 2021; accepted October 8, 2021.

Address correspondence to Frédérique Vanermen, M.D., Emmanuel Vierinlaan3.03 -8300 Knokke - Belgium.E-mail: Frederique.vanermen@ gmail.com

(C) 2021 THE AUTHORS. Published by Elsevier Inc. on behalf of the Arthroscopy Association of North America. This is an open access article under the CC BY-NC-ND license (http://creativecommons.org/licenses/by-nc-nd/4.0/). 2666-061X/21204

https://doi.org/10.1016/j.asmr.2021.10.002 piriformis muscle, which can extend down the lower limb and causes difficulty walking; (3) improvement with rest or by raising or giving traction to the affected limb; (4) palpation of a soft mass on the piriformis muscle; (5) straight leg raise sign; and (6) gluteal atrophy.

Historically, the term piriformis syndrome has been used to describe multiple entities with sciatic nerve compression within the subgluteal region, although in many cases the piriformis muscle was not the compressing force. Because multiple structures can compress the sciatic nerve within the subgluteal space, nowadays the term deep gluteal syndrome (DGS) is more frequently used to describe sciatic nerve entrapment within the subgluteal region, with PS being a subtype where the piriformis muscle is the compressing entity.

Anatomically, the subgluteal space is located between the middle and deep gluteal aponeurotic layers. The gluteus maximus serves as posterior border, the linea aspera and lateral fusion of the middle and deep gluteal aponeurosis layers as the lateral border, the posterior side of the femoral neck and both trochanters function as the anterior border, and the greater and minor sciatic foramina as the medial border. Inferiorly, the subgluteal space continues into the posterior thigh. Within 
this region, apart from the piriformis muscle, the superior and inferior gemellus muscle, the obturator internus muscle, and the quadratus femorus muscle also are located. ${ }^{4,5}$

Clinical presentation of PS is often pain in the gluteal region, aggravated when in a seating position with the inability to sit for a longer period of time, with or without pain radiating to the posterior thigh. Sometimes paresthesia is present as well. A history of trauma of the buttock is often present. ${ }^{6}$

Diagnosis is to the utmost extent based on a thorough clinical and physical examination, whereas the sensitivity and specificity of technical investigations such as radiographs, magnetic resonance imaging (MRI), magnetic resonance arthrography, and electromyography (EMG) are less effective in the diagnostic process than in other hip pathologies. ${ }^{6-8}$ PS is often difficult to diagnose, as the clinical presentation is rather vague and most clinical symptoms overlap with lumbar and other intra- or extra-articular hip pathologies, and therefore it is still underdiagnosed. ${ }^{5}$

Initial treatment for PS consists of physiotherapy regimens with stretching and muscle strengthening with or without an extra-articular injection with steroids or a local anesthetic. ${ }^{2,5,9}$ A surgical decompression and release of the nerve is considered after a failed conservative therapy of at least 3 months. Both open and endoscopic techniques have been described. ${ }^{10-14}$ Currently, the endoscopic approach is favored, as it is less invasive, offers a better view of the surrounding tissues, and has a lower infection rate in comparison with the open approach. . $^{, 14,15}$

In the literature, studies reporting on the endoscopic release specifically of PS are varied and most are case reports or small numbered case series. ${ }^{14,16,17}$ Ilizaliturri et al. ${ }^{12}$ published their results of endoscopic release in 14 patients with excellent or good results in all after 2 years. In recent years, several retrospective cohort studies such as Ham et al., ${ }^{6}$ Park et al., ${ }^{8}$ and AguileraBohorquez et al. ${ }^{10}$ have reported on the endoscopic treatment of DGS with good clinical results. As they mainly discuss the endoscopic treatment of DGS and only part of their population was diagnosed with the piriformis muscle as the compressive force needing a piriformis release, it remains difficult to compare.

The purpose of this study was to survey the clinical satisfaction of patients who have had an endoscopic piriformis release with a sciatic nerve neurolysis. We hypothesized that, in patients with refractory symptoms and a failed conservative treatment of PS, endoscopic piriformis release would provide improvement in clinical satisfaction and pain scores.

\section{Methods}

We retrospectively identified patients diagnosed with PS who were treated with an endoscopic release between 2013 and 2018. All patients had a minimal follow up of 1 year and were contacted for a telephone survey in 2019, whereby the rate of satisfaction and pain were questioned with the help of the Benson surgery scale (Table 1$)^{14}$ and visual analog scale (VAS) score, respectively. Also, patients were asked if, with the given knowledge, they would choose again to undergo an operative release. Approval of all patients was obtained before survey. Data and materials were obtained from the patient registration program at the Orthopaedic Centre Antwerp (ORTHOCA). The datasets used and/or analyzed during the current study are from corresponding author on reasonable request

All patients had a standardized diagnostic work-up (Fig 1) before being planned for a surgical release. Patient history, clinical presentation, and clinical examination were obtained according to Martin et al. ${ }^{18} \mathrm{~A}$ suggestive clinical presentation included (1) walking pain; (2) pain in a seated position, especially inability to sit for more than 30 minutes; (3) radicular pain in the lower limb with or without accompanying backpain; and (4) paresthesia.

Clinical examination was conducted in a standardized manner which included, apart from the standard hip and lower back examination, (1) palpation of the sciatic notch; (2) FAIR (flexion, adduction, internal rotation) test; (3) straight leg raise test; (4) seated piriformis test; (5) Pace sign; and (6) Freiberg's maneuver., ${ }^{71}$ A test was considered positive when the patient perceived a recognizable pain.

During the diagnostic process, patients underwent the following technical investigations: a radiograph (anteroposterior view) of the pelvis and hip and an MRI of the pelvis and hip to identify the trajectory of the sciatic nerve and rule out possible intrapelvic compression, and, an EMG with PS protocol (H-reflex latency between the hip in the neutral position and in FAIR position as described by Michel et al. ${ }^{19}$ and Fishman et al. $\left.{ }^{16,20}\right)$. All EMG investigations were done by the same team of physiatrists specializing in hip and lower limb pathology.

Table 1. The Symptom Rating Scale by Benson and Schutzer $^{14}$

\begin{tabular}{|c|c|}
\hline Outcome & Symptoms \\
\hline Excellent & $\begin{array}{l}\text { No pain with prolonged period of sitting (>30 min), } \\
\text { strenuous activity, bending, twisting, stairs, rapid } \\
\text { walking, jogging. }\end{array}$ \\
\hline Good & $\begin{array}{l}\text { No pain with short periods of sitting }(<30 \mathrm{~min}) \text { or daily } \\
\text { activities or mild pain with prolonged period of sitting or } \\
\text { strenuous activity. }\end{array}$ \\
\hline Fair & $\begin{array}{l}\text { Occasional mild pain with short periods of sitting or } \\
\text { normal daily activities or moderate pain with prolonged } \\
\text { sitting or strenuous activity }\end{array}$ \\
\hline Poor & $\begin{array}{l}\text { Severe pain with short periods of sitting or normal daily } \\
\text { activities little change from preoperative level of pain } \\
\text { associated with sciatic nerve }\end{array}$ \\
\hline
\end{tabular}



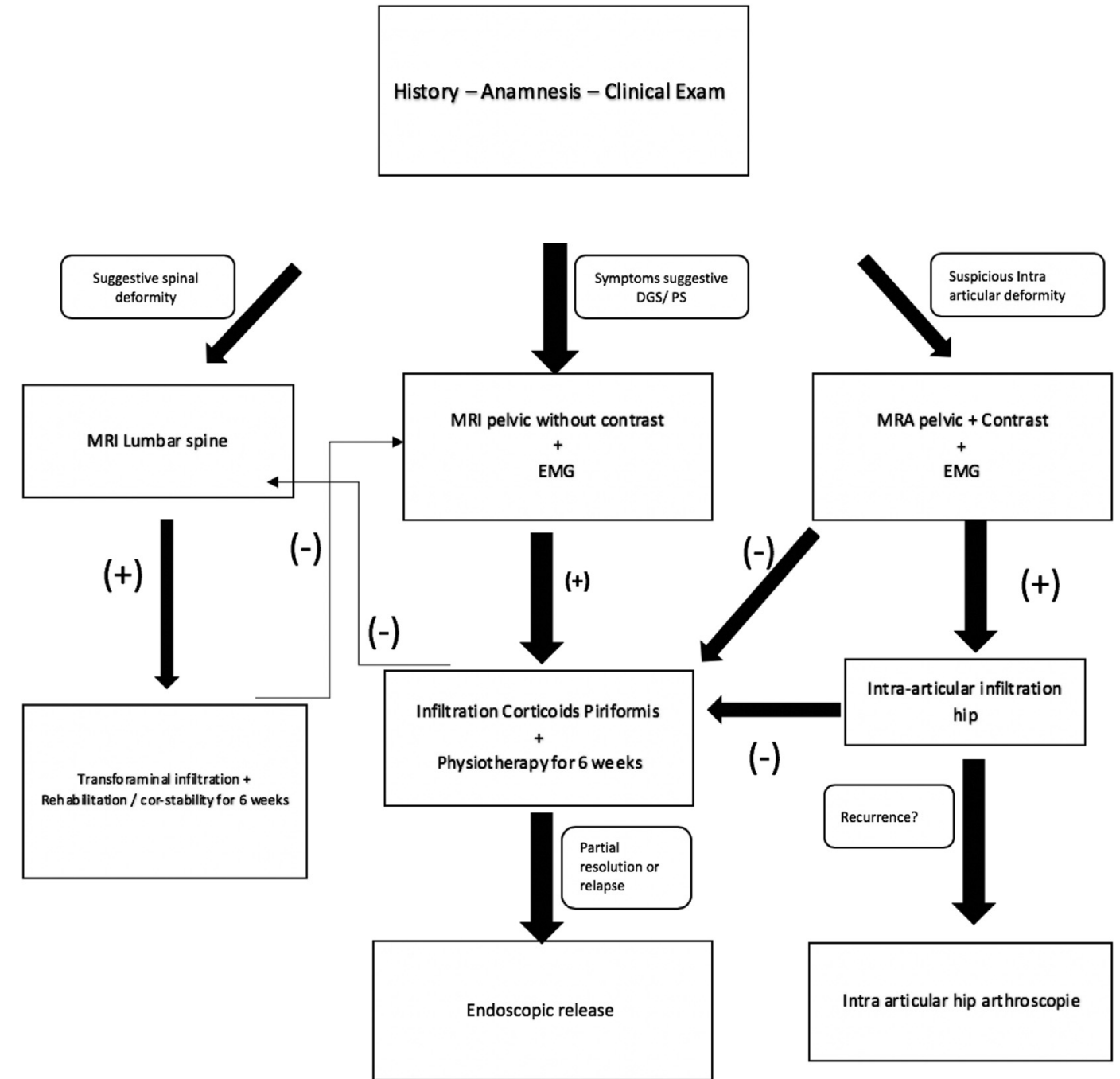

Fig 1. Diagnostic decision protocol. (DGS, deep gluteal syndrome; EMG, electromyography; MRA, magnetic resonance arthrography; MRI, magnetic resonance imaging; PS, piriformis syndrome.)

Initial treatment consisted of a minimum of 3 months of physical therapy (consisting among other of stretching of the gluteal muscles, external rotators of the hip, and hamstrings) combined with an ultrasoundguided injection with steroids. All surgeries were performed by a single surgeon who had more than 20 years of experience with hip arthroscopy.

Analysis was carried out using SPSS 23.0 software (IBM Corp., Armonk, NY). The Shapiro-Wilk test was used to assess normality of distribution. The Wilcoxon signed rank was used to compare pre- and postoperative data (VAS scores). The Mann-Whitney $U$ test was used for continuous variables with non-normal distribution. Significance was defined as a $P$ value $\leq .05$.

The study was approved by the local hospital ethics committee and local Research Ethics Board operating according to the International Council for Harmonisation of Technical Requirements for Pharmaceuticals for Human Use/Good Clinical Practice guidelines. Informed consent was obtained by all the patients as required by the ethics committees.

\section{Surgical Procedure}

The surgical procedure was performed with the patient in a lateral decubitus position on a regular operating table without traction (Fig $2 \mathrm{~A}$ and $\mathrm{B}$ ). The greater trochanter was palpated and marked together with the standard posterolateral and lateral accessory portal. We used an arthroscopic camera of $30^{\circ}$. After making the arthroscopic portals, the trochanteric bursa was identified and resected with the aid of an electrocautery and shaver. After the bursectomy, the external rotators and the piriformis muscle was exposed, followed by a careful medial dissection to identify the sciatic nerve and the piriformis tendon at its course just proximal to the short external rotators. With the use of the electrocautery and shaver, the sciatic nerve was carefully 

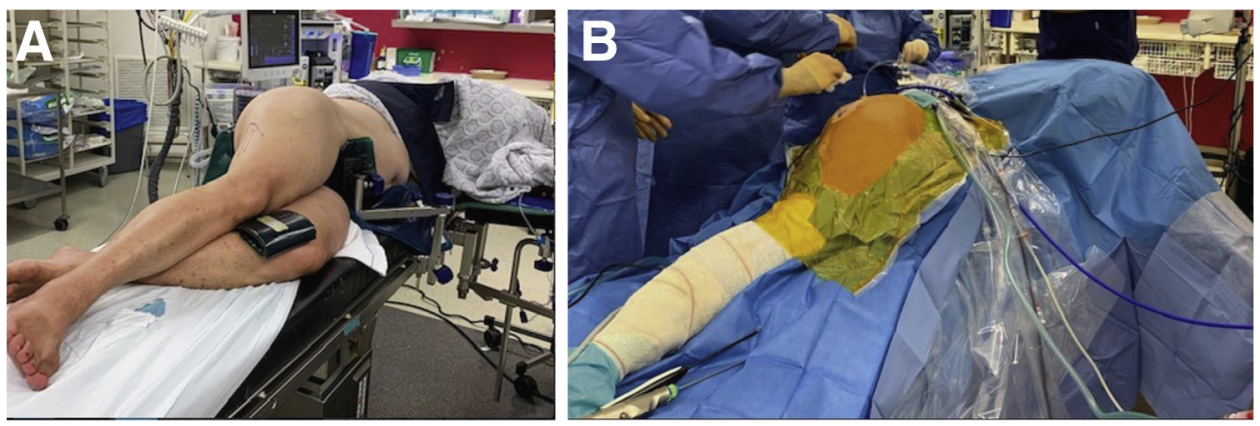

Fig 2. (A) Installation in lateral decubitus before draping of a right hip with protection of pressure areas. (B) Installation after sterile draping. The ipsilateral leg is draped separately to allow rotation during surgery.

further exposed with dissection of adhesive bands proximally and distally. The proximal and distal portion of the nerve were released to allow complete visualization of the course of the nerve up until its pelvic entrance. Once the nerve was adequately liberated, the piriformis was released off its attachment at the piriformis fossa and the greater trochanter. Care was taken to carry out a complete release to reduce the risk of recurrence of sciatic nerve entrapment by the piriformis muscle.

Postoperatively, immediate mobilization was permitted, with restrictions on deep flexion and internal rotation and patients were put on partial weight-bearing for 3 weeks. After 3 weeks, full weight-bearing was allowed and physical therapy with stretching and a rehabilitation program for hip, pelvis, and lower back was started. Patients were seen 3 weeks (wound control), 3 months, and 6 months postoperatively and were contacted by one of the authors for a telephone survey in the beginning of 2019 .

\section{Results}

In total, 45 hips in 44 patients were included, 16 male and 28 female, with an average age of 45.8 years $( \pm 14.8)$. The average postoperative time was 3.7 years. Average duration of symptoms at the first presentation was 34 months (range 16-54 months). In total, 36 patients (37 hips; $82.2 \%$ ) had an EMG that showed sciatic compression around the piriformis muscle. The average VAS pain score preoperatively was $7.4( \pm 0.8)$, and the VAS pain scores at 3 weeks, 3 months, 6 months, and at the control by telephone survey were $4.1( \pm 2.1), 2.5$ ( \pm $2.4), 1.9( \pm 2.4)$, and $2.1( \pm 2.6)$, respectively. Using the Wilcoxon signed-rank test, we found there was a significant improvement of VAS score with $P<.001$. Age, sex, and lateralization were proven not be confounding variables. The Benson operative scale outcomes are summarized in Table 2. At the time of the telephone survey, 24 cases $(53.4 \%)$ rated the result as excellent, $10(22.2 \%)$ as good, $3(6.7 \%)$ as fair, and $8(17.7 \%)$ as poor. In total, 33 patients answered affirmative on the question if they would undergo the procedure again. Of the 8 patients who had a poor result, 2 patients marked on the first 3 control's the result as excellent with a VAS score of 2, 2, and 1 after 3 weeks, 3 and 6 months postoperative, respectively, but both relapsed after 2 years and had the same complaints as preoperatively at the time of the telephone survey. The other 6 patients with a poor result had an average age of 55.1 years (range 34-69 years) and remained symptomatic during the whole follow-up period with an average VAS score of 7.5 on the 3-month control, 5.7 on the 6-month control and 6,4 during the telephone survey. One patient went on to have a total hip replacement with total resolution of the pain. One patient had a redo sacroiliac

Table 2. Overview of Results

\begin{tabular}{|c|c|}
\hline Results & Data ( $\mathrm{n}=45$ hips in 44 Patients) \\
\hline \multicolumn{2}{|l|}{ Sex } \\
\hline Male & $16 / 45$ \\
\hline Female & $29 / 45$ \\
\hline Age, y (standard deviation) & $45.8( \pm 14.8)$ \\
\hline \multicolumn{2}{|l|}{ Operative side } \\
\hline Left & $21 / 45$ \\
\hline Right & $24 / 45$ \\
\hline $\begin{array}{l}\text { Duration of symptoms, mo } \\
\text { (range) }\end{array}$ & $34(16-54)$ \\
\hline \multicolumn{2}{|l|}{ Pain evaluation } \\
\hline \multicolumn{2}{|l|}{ Visual analog score } \\
\hline Preoperative & $7.4( \pm 0.81)$ \\
\hline 3 weeks' postoperative & $4.1( \pm 2.1)$ \\
\hline 3 months' postoperative & $2.6( \pm 2.5)$ \\
\hline 6 months' postoperative & $1.9( \pm 2.4)$ \\
\hline Telephone survey & $2.2( \pm 2.6)$ \\
\hline \multicolumn{2}{|l|}{$\begin{array}{l}\text { Benson surgical outcomes, \% } \\
\text { (telephone survey) }\end{array}$} \\
\hline Excellent & $24(53.3 \%)$ \\
\hline Good & $10(22.2 \%)$ \\
\hline Fair & $3(6.7 \%)$ \\
\hline Poor & $8(17.8 \%)$ \\
\hline \multicolumn{2}{|l|}{ Complications, \% } \\
\hline Superficial wound infection & $3(6.7 \%)$ \\
\hline \multicolumn{2}{|l|}{$\begin{array}{l}\text { Would choose again for } \\
\text { operative treatment ( } \% \text { of } \\
44 \text { patients) }\end{array}$} \\
\hline Yes & $32(72.7 \%)$ \\
\hline No & $8(18.2 \%)$ \\
\hline No answer & $4(9 \%)$ \\
\hline
\end{tabular}


infiltration followed by a sacroiliac denervation with a good result. Two patients were referred to the spine unit for further investigations and received a transforaminal infiltration with improvement of the complaints. In the 2 other patients, no other origin of the complaints could be found thus far. No revision surgeries were performed. Three patients were treated for a local wound infection with oral antibiotics (coamoxiclav-3 times $875 \mathrm{mg}$ per day for 7 days) and continued wound care and went on to heal without any further problems.

In 23 of the 24 cases $(95.8 \%)$ who reported an excellent result and all 10 of the cases reporting a good outcome on the Benson outcome scale showed signs of sciatic compression on EMG testing. Two patients with signs of compression on EMG reported a fair result at follow-up and 1 patient with a positive EMG test had an initial excellent result but relapsed. One patient, although EMG showed signs of compression of the sciatic nerve, had a poor result.

During the telephone survey, 6 patients (13.3\%) remarked that, although they had a good reduction of pain, they never regained full power of their hip muscles, even after multiple sessions of physiotherapy, whereas 25 patients $(55.6 \%)$ needed 5 to 7 months and up to 30 sessions of physiotherapy to regain full strength. Demographic data and results are summarized in Table 2.

\section{Discussion}

PS is a muscular disorder occasioned by the sciatic nerve becoming compressed by the piriformis muscle in the subgluteal space causing sciatic-type pain with often the inability to sit for a longer period of time and paresthesia in the buttock along the sciatic nerve pathway into the thigh and leg. ${ }^{19}$

In the literature, studies reporting on the endoscopic release of PS are varied, and most are case reports or small numbered case series. ${ }^{14,16,17}$ Our series with 45 patients is one of the largest published to date.

In this study, all patients were treated for PS with a release of the piriformis tendon and a decompression of the sciatic nerve. Diagnosis was made after a standardized workup protocol (Fig 1) containing a thorough history, physical examination, and routine pelvis radiography, together with an MRI of the pelvis/hip and EMG. Often, it remains a difficult diagnosis due to the vague clinical symptoms and the absence of validated clinical criteria. ${ }^{2}$

In our cohort, although we have a well-established diagnostic algorithm in place, 3 patients were wrongly diagnosed with PS, ending up with a poor result. The mean preoperative and postoperative VAS scores were 7.4. $( \pm 0.8)$ and $2.1( \pm 2.6)$, respectively. This is comparable with outcomes scores reported by Ilizaliturri et al. ${ }^{12}$ and Hun et al., ${ }^{6}$ who reported postoperative VAS scores of $1.86( \pm 0.83)$ and $2.5( \pm 1.5)$, respectively.

At final follow up, 34 patients $(75,6 \%)$ reported an excellent or good clinical outcome according to the Benson surgical scale. This is slightly lower than the reported scores by Ilizaliturri et al., ${ }^{12}$ Park et al., ${ }^{8}$ and Hun et al., ${ }^{6}$ who reported excellent or good clinical outcomes in, respectively, 14 of $15(93.3 \%), 53$ of 60 $(88.3 \%)$, and 21 of $24(87.5 \%)$ patients. Our clinical results were reported at a mean follow-up of 3.7 years, whereas for Ilizaliturri et al. ${ }^{12}$ and Hun et al., ${ }^{6}$ their clinical outcomes reported at 2 years' and 1-year postoperative respectively.

In our center, MRI of the pelvis is used to evaluate for the presence of anatomic variations of the piriformis muscle and sciatic nerve as described by Beaton and Anson, ${ }^{22}$ and to screen for any space-occupying lesions that can generate compression on the sciatic nerve ${ }^{22}$ According to Martin et al., ${ }^{18}$ MRI usually doesn't provide sufficient information to confirm sciatic nerve compression within the deep gluteal space, whereas others such as Filler et al. ${ }^{9}$ describe diagnostic efficacy of MR neurography with a $93 \%$ specificity and $64 \%$ sensitivity for piriformis muscle asymmetry and sciatic nerve hypersensitivity at the sciatic notch. In our center, MRI of the pelvis is used to evaluate for the presence of anatomic variations of the piriformis muscle and sciatic nerve as described by Beaton and Anson ${ }^{22}$ and to screen for any space-occupying lesions that can generate compression on the sciatic nerve.

EMG is known to be a validated tool in the workup for PS by looking for the H-reflex latency between the hip in neutral position and with the hip in flexion, adduction, and internally rotated. ${ }^{16,19,20}$ Although a few authors describe the use of an EMG in some patients as a diagnostic aid, it is not often routinely used in the workup. ${ }^{16,19,20}$ In our practice, an EMG is routinely obtained for all patients with suspicion of PS by a team of rehabilitation specialists. In 33 of $34(97 \%)$ cases with excellent or good results, the EMG was positive for extra-lumbar sciatic nerve compression around the piriformis muscle.

\section{Limitations}

This study is not without limitations: first, it is a retrospective cohort study of a single-surgeon series with a relatively limited number of cases. Nonetheless, it is one of the larger cohorts in comparison with comparable studies. Second, the latest follow up consisted of a telephone survey and not a face-to-face appointment, which limits the possibility of a clinical examination.

\section{Conclusions}

Once a diagnosis is made, the endoscopic release of the piriformis tendon results in significant 
improvement in Pain VAS scores for patients with refractory symptoms despite conservative treatment.

\section{References}

1. Jennes B, Bogaerts S, Bruyninckx F, Van Wambeke P, Lysens R. Het piriformissyndroom: Overzicht van de recente literatuur. Tijdschr Geneeskd 2013;69:275-285 [in Dutch].

2. Kay J, de Sa D, Morrison L, et al. Surgical management of deep gluteal syndrome causing sciatic nerve entrapment: A systematic review. Arthroscopy 2017;33:2263-2278.el.

3. Robinson DR. Pyriformis syndrome in relation to sciatic pain. Am J Surg 1947;73:355-358.

4. Carro LP, Hernando MF, Cerezal L, Navarro IS, Fernandez AA, Castillo AO. Deep gluteal space problems: Piriformis syndrome, ischiofemoral impingement and sciatic nerve release. Muscles Ligaments Tendons J 2016;6:384-396.

5. Hernando MF, Cerezal L, Pérez-Carro L, Abascal F, Canga A. Deep gluteal syndrome: Anatomy, imaging, and management of sciatic nerve entrapments in the subgluteal space. Skeletal Radiol 2015;44:919-934.

6. Ham DH, Chung WC, Jung DU. Effectiveness of endoscopic sciatic nerve decompression for the treatment of deep gluteal syndrome. Hip Pelvis 2018;30:29.

7. Martin HD, Kivlan BR, Palmer IJ, Martin RRL. Diagnostic accuracy of clinical tests for sciatic nerve entrapment in the gluteal region. Knee Surg Sport Traumatol Arthrosc 2014;22:882-888.

8. Park MS, Yoon SJ, Jung SY, Kim SH. Clinical results of endoscopic sciatic nerve decompression for deep gluteal syndrome: Mean 2-year follow-up. BMC Musculoskelet Disord 2016;17:1-7.

9. Filler AG, Haynes J, Jordan SE, et al. Sciatica of nondisc origin and piriformis syndrome: diagnosis by magnetic resonance neurography and interventional magnetic resonance imaging with outcome study of resulting treatment. J Neurosurg Spine 2005;2:99-115.

10. Aguilera-Bohorquez B, Cardozo O, Brugiatti M, Cantor E, Valdivia N. Endoscopic treatment of sciatic nerve entrapment in deep gluteal syndrome: Clinical results. Rev Española Cirugía Ortopédica y Traumatol (English Ed) 2018;62:322-327.

11. Martin HD, Hatem M, Palmer IJ. Endoscopic sciatic nerve decompression: Operative technique. Oper Tech Sports Med 2012:20:325-332.

12. Ilizaliturri VM, Arriaga R, Villalobos FE, Suarez-Ahedo C. Endoscopic release of the piriformis tendon and sciatic nerve exploration. J Hip Preserv Surg 2018;5:301-306.

13. Kullander K, Klein R. Piriformis muscle syndrome in 19 patients treated by tenotomy-A 1- to 16-year follow-up study. Int Orthop 2002;26:101-103.

14. Benson ER, Schutzer SF. Posttraumatic piriformis syndrome: Diagnosis and results of operative treatment. J Bone Joint Surg Am 1999;81:941-949.

15. Dezawa A, Kusano S, Miki H. Arthroscopic release of the piriformis muscle under local anesthesia for piriformis syndrome. Arthroscopy 2003; 19:554-557.

16. Fishman LM, Dombi GW, Michaelsen C, et al. Piriformis syndrome: Diagnosis, treatment, and outcome-A 10year study. Arch Phys Med Rehabil 2002;83:295-301.

17. Knudsen JS, McConkey MO, Brick MJ. Endoscopic sciatic neurolysis. Arthrosc Tech 2015;4:e353-e358.

18. Martin HD, Shears SA, Johnson JC, Smathers AM, Palmer IJ. The endoscopic treatment of sciatic nerve entrapment/deep gluteal syndrome. Arthroscopy 2011;27: 172-181.

19. Michel F, Decavel P, Toussirot E, et al. Syndrome du muscle piriforme: Critères diagnostiques et traitement à propos d'une série monocentrique de 250 patients. Ann Phys Rehabil Med 2013;56:371-383.

20. Fishman LM, Zybert PA. Electrophysiologic evidence of piriformis syndrome. Phys Med Rehabil 1992;73:359-364.

21. Hopayian K, Song F, Riera R, Sambandan S. The clinical features of the piriformis syndrome: A systematic review. Eur Spine J 2010;19:2095-2109.

22. Beaton L, Anson B. The relation of the sciatic nerve and of its subdivisions to the piriformis muscle. Anat Rec 1937;70: 1-5. 